\title{
Extending the Longevity of Fluorescence-Based Sensor Arrays Using Adaptive Exposure
}

Supporting Information

Sandra Bencic-Nagale and David R. Walt

\begin{abstract}
Materials. Nile Red dye was purchased from Aldrich (Milwaukee, WI). SNAFL dye with a succinimidyl ester functional group was purchased from Molecular Probes (Eugene, OR). Platinum EPS C4 and IBSil $\left(\mathrm{NH}_{2}\right)$ beads were purchased as dry stocks from Alltech (Deerfield, IL) and Phenomenex (Torrance, CA), respectively. Chirex 3012 and Selectosil (SCX) beads were retrieved from Phenomenex liquid chromatography columns. Polished and etched optical fiber bundles with approximately 24,000 $4.5 \mu \mathrm{m}-$ diameter wells were obtained from Illumina (San Diego, CA).
\end{abstract}

Sensor Fabrication. Silica beads were removed from chromatography columns, washed with toluene, and allowed to dry overnight at $60^{\circ} \mathrm{C}$. The preparation of dye-coated microbead sensors required either adsorption of Nile Red or covalent attachment of SNAFL dye molecules onto the surface of the beads. Dye solutions were combined with bead stocks $(0.1 \mathrm{~mL}$ dye solution per $\mathrm{mg}$ of beads) in 4-mL glass vials and mixed for 1 hour. Table S1 lists the bead material specifications and the types and concentrations of dyes used. After mixing, bead suspensions were filtered and rinsed with either DMF or toluene. The Alltech, Chirex, and Snafl sensor stocks were dried at $60^{\circ} \mathrm{C}$ for 16 hours, and $\mathrm{Sel}$ beads were dried for 3 hours. Dry sensor stocks were stored in a dessicator until use. A mixture of equal weights of these sensor stocks was then prepared for the fabrication of randomized bead sensor arrays. Arrays with high sensor packing density and uniform distribution across the etched face of the fiber were prepared by gently tapping the etched fiber ends with the dry bead mixture. Excess beads were then wiped off the surface of the fiber bundle with a dust-free Texwipe clean room swab (Fisher Scientific). 
Sensor Preconditioning. After acquisition of responses used in array registration, the sensors were preconditioned with a 10-min saturated water vapor purge, followed by a 50-min air purge. Water vapor and air were delivered to the sensors at $200 \mathrm{~mL} / \mathrm{min}$. The preconditioning protocol was developed because irreproducibility of Snafl sensor responses was observed in the beginning of array usage when new arrays, prepared from the dry sensor stock, were positioned on the imaging setup. Our control experiments indicated that Snafl sensors responded reproducibly after they were humidified and then immediately dried in air. The emission bands of the SNAFL-coated microbead sensors shifted and their intensities changed after the preconditioning step, indicating that the surface $\mathrm{pH}$ might have affected the dye's fluorescence properties. The first set of vapor responses acquired with sensors on the first sub-section at 2.0 ND was also discarded because some of the Snafl responses to vapors were still inconsistent. The Snafl responses acquired during the remainder of the longevity experiment were reproducible and were therefore included in the data analysis.

Table S1. Sensor materials and fabrication conditions

Sensor Name Bead Type, Diameter Surface Functionality

$\begin{array}{cc}\text { Alltech } & \text { Alltech EPS C4, } 5 \mathrm{~mm} \\ \text { Chirex } & \text { Chirex 3012, 5 mm } \\ \text { Sel } & \text { Selectosil (SCX), } 5 \mathrm{~mm} \\ \text { Snafl } & \text { IBSil }\left(\mathrm{NH}_{2}\right), 3 \mathrm{~mm}\end{array}$

Alltech Snafl

$-\mathrm{C}_{4} \mathrm{H}_{10}$

(R)-phenylglycineand 3,5-dinitroaniline urea linkage cation exchange

$-\mathrm{NH}_{2}$
Dye (Conc.)

Nile Red $(0.5 \mathrm{mg} / \mathrm{mL}$ toluene $)$

Nile Red (0.5 mg/mL toluene)

Nile Red $(0.01 \mathrm{mg} / \mathrm{mL}$ toluene $)$

SNAFL (0.2 mg/mL DMF) 